TITLE:

\title{
Kinetic analysis of ion incident angle distribution on a plasma- facing wall
}

$\operatorname{AUTHOR}(S)$ :

Kawamura, G.; Fukuyama, Atsushi; Tomita, Y.

\section{CITATION:}

Kawamura, G....[et al]. Kinetic analysis of ion incident angle distribution on a plasmafacing wall. Journal of Nuclear Materials 2009, 390-391: 172-174

\section{ISSUE DATE:}

2009-06-15

URL:

http://hdl.handle.net/2433/123370

\section{RIGHT:}

Copyright (C) 2009 Elsevier B.V.; この論文は出版社版でありません。引 用の際には出版社版をご確認ご利用ください。; This is not the published version. Please cite only the published version. 


\title{
Kinetic analysis of ion incident angle distribution on a plasma-facing wall
}

\author{
G. Kawamura ${ }^{\mathrm{a}}$, A. Fukuyama ${ }^{\mathrm{b}}$ and Y. Tomita ${ }^{\mathrm{a}}$ \\ ${ }^{a}$ National Institute for Fusion Science \\ 322-6 Oroshi-cho Toki-shi Gifu 509-5292, Japan \\ ${ }^{\mathrm{b}}$ Graduate School of Engineering, Kyoto University \\ Yoshida-Honmachi Sakyo-ku Kyoto 606-8501, Japan
}

\begin{abstract}
A one-dimensional particle simulation model of a magnetic presheath was presented and a simulation study was made to reveal the effect of the magnetic field on the ion incident angle distribution on a plasma-facing surface of a fusion device. Two kinds of plasma source due to the cross-field diffusion in the scrape-off layer and the ionization in the collisional presheath are considered to model the velocity distribution function at the entrance of the magnetic presheath. The dependence of the ion incident angle distribution was examined and a fitting form of the energy flux to the surface was obtained as a function of the incident angle and the magnetic field. A transition of the incident angle distribution was found. Dependences of the incident angle on the magnetic field strength vanishes when the magnetic field becomes weaker or stronger than threshold values. In the transition, when the magnetic field approaches parallel to the surface, incident angles become large.
\end{abstract}

\section{Introduction}

Understanding of the plasma-surface interaction has been recognized as a crucial physical and engineering issue in fusion devices. Optimizations of the plasma parameters, magnetic field and geometry of the plasma-facing wall are necessary to control the impurity production and transport. One of the key physical factors is the sputtering from the wall material by the ion impacts. The sputtering yield depends on the energy flux of the impact ions and the incident angle [1].

The plasma in the scrape-off layer (SOL) is divided into three region [2]; collisional presheath (CP), magnetic presheath (MP) and Debye sheath (DS). The plasma in the CP region can be described well by the fluid quantities such as density, velocity and temperature [3], while the MP and DS regions require a kinetic description and a particle simulation is necessary. Although the precedent studies of the incident angle are found in Refs. [4,5], they gave the parameter dependence of the averaged incident angle only. Our work was motivated by the necessity of more detailed analyses including the distribution of the incident angle to understand plasma-surface interaction deeply.

We present a one dimensional particle simulation model of MP and DS regions in Sec. 2. A velocity distribution function which models characteristics of the plasma source in the SOL is introduced. In Sec. 3, the incident angle distribution of the ions hitting the wall surface is examined and a fitting form is obtained from simulation results for various magnetic field strengths and directions. Conclusions are presented in Sec. 4.

\section{Simulation model}

In order to examine the dependences of the ion incident angle distribution to the wall surface on the magnetic field, we employ the particle-in-cell (PIC) simulation method. The coordinate system used here is illustrated in Fig. 1. The $x$ axis is taken to be normal to the wall surface and the system length, or the position of the wall, is denoted by $L$. The plasma profiles such as density $n$ and potential $\phi$ are assumed to vary only in the $x$ direction. We have developed a PIC code to solve the equations of motion and Poisson's equation self-consistently. The magnetic field is assumed to be uniform and its direction is specified by the angle $\varphi$ in the $x-y$ plane, or $\mathbf{B}=B \cos \varphi \hat{\mathbf{x}}+B \sin \varphi \hat{\mathbf{y}}$. The incident angle of the ion is denoted by $\theta$, i.e. $\cos \theta=\hat{\mathbf{x}} \cdot \mathbf{v} / v$. Here velocity of each particle hitting the wall surface was denoted by $\mathbf{v}$. Although a velocity requires two angles to determine its direction, we use only the vertical angle because the azimuthal angle does not play a role in isotropic materials. The wall is perfectly absorbing 


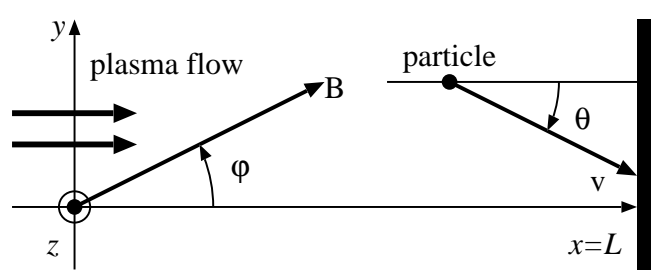

FIG. 1: Coordinate system used in the PIC simulation.

and electrically floating. Therefore, the electric field at $x=L$ is determined by the Gauss' theorem from the charge on the wall. The system length $L$ is taken to be several times longer than the ion Larmor radius to allow the MP layer [6]. Since the mean-free-path of the collisions between ions and neutrals are much longer than the Larmor radius, particle source is not included in the simulation. Instead, we place a source boundary at $x=0$, where the velocity distribution is fixed to a given function.

The determination of a velocity distribution at the entrance of MP is an essential issue for PIC simulation of sheath. However, there is no commonly accepted means to determine it in such an openended plasma. In this work, we use the following equation as an example of the distribution function:

$$
f_{0}\left(v_{\|}, v_{\perp}, \theta\right)=n_{0} \sqrt{\frac{2}{\pi^{3}}}\left(\frac{m_{\mathrm{i}}}{Z T_{\mathrm{e}}}\right)^{5 / 2} v_{\|}^{2} \exp \left(-\frac{m_{\mathrm{i}} v_{\|}^{2}}{2 Z T_{\mathrm{e}}}-\frac{m_{\mathrm{i}} v_{\perp}^{2}}{Z T_{\mathrm{e}}}\right),
$$

where the density at $x=0$, perpendicular velocity and gyro-phase of a particle were denoted by $n_{0}$, $v_{\perp}$ and $\theta$ respectively. The function has two characteristics corresponding to ionization component transported from the collisional presheath and cross-field diffusion component from upstream SOL. The former is relatively low energy and proportional to $v_{\|}^{2}$ at $v_{\|} \sim 0[8,9]$. The latter is approximately Maxwellian with acceleration by the CP potential drop in front of MP [3,10,11]. The distribution function given by Eq. (1) is consistent to a PIC simulation result [7]. Eq. (1) also satisfies the generalized Bohm criterion [9] with the equality, i.e. $\left\langle v^{-2}\right\rangle=m_{\mathrm{i}} / Z T_{\mathrm{e}}$. The brackets represents the average over the velocity space. The electron temperature, ion mass and charge were denoted by $T_{\mathrm{e}}, m_{\mathrm{i}}$ and $Z e$. By integrating Eq. (1) we obtain the plasma velocity and temperature; $u_{\mathrm{i}}=\sqrt{8 Z T_{\mathrm{e}} / \pi m_{\mathrm{i}}} \simeq 1.6 c_{\mathrm{s} 0}$ and $T_{\mathrm{i}}=(3-8 / \pi) Z T_{\mathrm{e}} \simeq 0.45 Z T_{\mathrm{e}}$. Here the cold ion sound speed was denoted by $c_{\mathrm{s} 0} \equiv \sqrt{Z T_{\mathrm{e}} / m_{\mathrm{i}}}$. Since the adiabatic ion sound speed is given by $c_{\mathrm{s}} \equiv \sqrt{\left(Z T_{\mathrm{e}}+3 T_{\mathrm{i}}\right) / m_{\mathrm{i}}} \simeq 1.5 c_{\mathrm{s} 0}$, the Bohm criterion is also satisfied. The detailed discussion on the determination of the distribution function will be presented in future publications.

\section{Simulation results and discussions}

In this section, we show PIC simulation results and examine the dependences of the ion incident angles to the wall surface on the magnetic field. Before that, we show the plasma profiles obtained in the particle simulation with various strength of the magnetic field. Figs. 2 (a) and (b) represent the profiles of the potential and the charge density for $Z=1$ and $m_{\mathrm{i}} / m_{\mathrm{e}}=1836$, i.e. hydrogen plasma. The four curves in each figure correspond to four different strength of the magnetic field; $B=0$ and $r_{\mathrm{L}} / \lambda_{\mathrm{De}}=1$, 2 and 4 . The thermal ion Larmor radius at $x=0$ was denoted by $r_{\mathrm{L}} \equiv \sqrt{T_{\mathrm{i} \perp} / m_{\mathrm{i}}} m_{\mathrm{i}} / Z e B$. The magnetic field direction is fixed to $B_{x} / B=1 / 16$ or $\varphi=86.4^{\circ}$. The fact that the quasi-neutrality is satisfied in the slope except for the thin area near the wall, $\Delta_{\mathrm{DS}} \sim 7 \lambda_{\mathrm{De}}$, indicates the formation of MP in front of DS. Although the boundary between MP and DS is not clear, the width of DS is almost independent of the magnetic field strength. The width of MP is roughly proportional to the thermal ion Larmor radius as Chodura found in Ref. [6].

The energy flux distributions for the magnetized plasma are shown in Fig. 3. We used the thermal ion Larmor radius of $r_{\mathrm{L}} / \lambda_{\mathrm{De}}=4$ here. The cross, plus and asterisk marks represent the simulation results for $B_{x} / B=15 / 16,1 / 2$ and $1 / 16$ respectively. In order to describe the characteristics of the profiles, we 


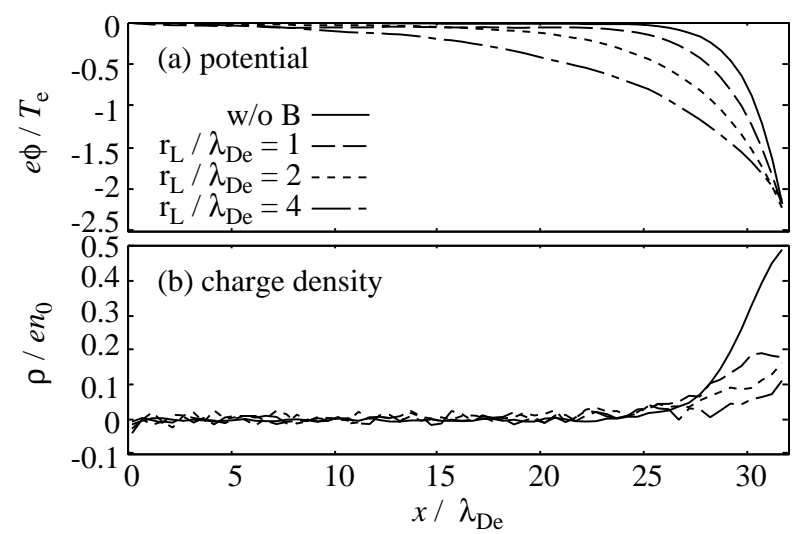

FIG. 2: Profiles of the potential and the charge density for different magnetic field strength; $B=0$ and $r_{\mathrm{L}} / \lambda_{\mathrm{De}}=1,2$ and 4 .

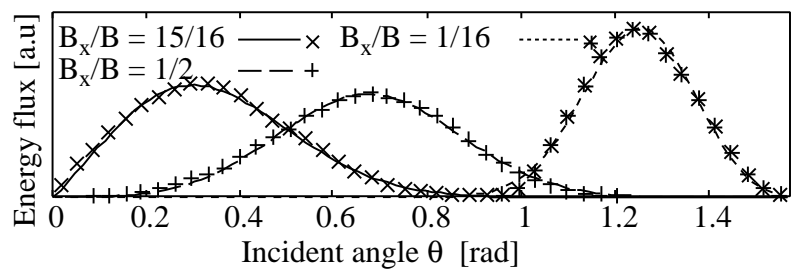

FIG. 3: Distribution of energy fluxes as functions of the ion incident angle. Simulation results $(\times,+$ and * marks) and fitting curves (solid, dashed and dotted curves) are plotted.

introduce a fitting function

$$
Q(\alpha, \beta, \gamma ; \theta)=\alpha \sin 2 \theta \exp \left[-\beta(\theta-\gamma)^{2}\right],
$$

where the parameter $\alpha, \beta$ and $\gamma$ are functions of the magnetic field strength and direction. The factor, $\sin 2 \theta \equiv 2 \sin \theta \cos \theta$, represents variations proportional to the solid angle, $\sin \theta$, and the normal component of the flux, $\cos \theta$. We obtained the best fitting parameters and plotted them as the solid, dashed and dotted curves in Fig. 3. The fitting curves agree with the simulation results quite well.

We examined the dependences of the parameters $\beta$ and $\gamma$ in Eq. (2) on the magnetic field. The parameter $\alpha$ is ignored in this work because it represents the normalization factor and can be obtained from the other parameters. We carried out the particle simulation for the following parameters; $r_{\mathrm{L}} / \lambda_{\mathrm{De}}=$ $1,2,4,8,16$ and 32 , and $B_{x} / B=1 / 16,1 / 8,1 / 4,1 / 2,3 / 4,7 / 8$ and $15 / 16 . r_{\mathrm{L}} / \lambda_{\mathrm{De}}=1 / 8-32$ and $B_{x} / B=$ $1 / 32-31 / 32$. Firstly we show the dependence of the parameter $\beta$ on the magnetic field in Fig. 4(a). The cross and plus marks represent $\beta$ for $r_{\mathrm{L}} / \lambda_{\mathrm{De}}=1$ and 32 respectively. Parameter $\beta$ corresponds to the width of the $\theta$ distribution. When the magnetic field is weak, $r_{\mathrm{L}} / \lambda_{\mathrm{De}} \gg 1$, the dependence of $\beta$ on the angle of the magnetic field $\varphi$ is weak except $\varphi \sim \pi / 2$. Two curves in Fig. 4(a) represent fitting functions given by $\beta=b_{1} \exp \left[b_{2} \varphi^{2} /\left(b_{3}-\varphi\right)\right]$. New parameter $b_{1}, b_{2}$ and $b_{3}$ are functions of only magnetic field strength. Our simulation results indicate that the first one, $b_{1}$, is almost constant. The other two are plotted in Figs. 4(b) and (c) respectively and we confirmed they are characterized by the hyperbolic tangent. That implies the incident angle distribution has a transition and changes only when the magnetic field strength is in the range of $0<\ln \left(r_{\mathrm{L}} / \lambda_{\mathrm{De}}\right)<2$.

Secondly we show the dependence of the parameter $\gamma$ on the magnetic field in Fig. 5(a). The cross and plus marks represent $\gamma$ for $r_{\mathrm{L}} / \lambda_{\mathrm{De}}=1$ and 32 respectively. Parameter $\gamma$ corresponds to the peak position of the $\theta$ distribution. When the magnetic field is nearly perpendicular to the surface normal, $\gamma$ does not depend on the magnetic field strength. Since the magnetic field restrict the acceleration of ions due to the electric field toward the wall, a strong magnetic field makes the incident angle large. Two fitting curves in Fig. 5(a) are given by $\gamma=(1-\cos \varphi) \pi / 2-\varphi^{2} \sqrt{\pi / 2-\varphi}\left[c_{1} \varphi-c_{2}(\pi / 2-\varphi)\right]$. The 

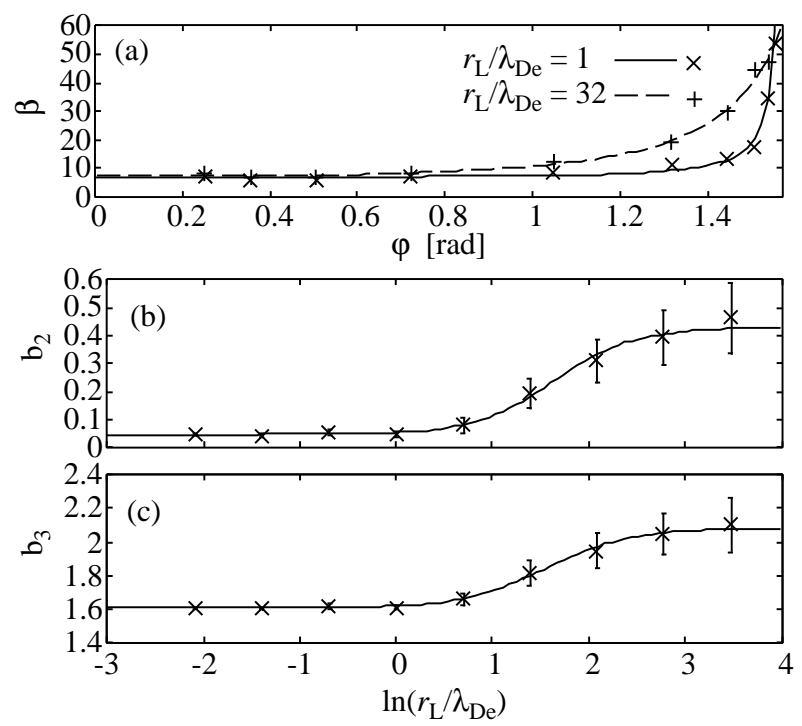

FIG. 4: Dependences of the parameters $\beta, b_{2}$ and $b_{3}$. Curves represent fitting functions.
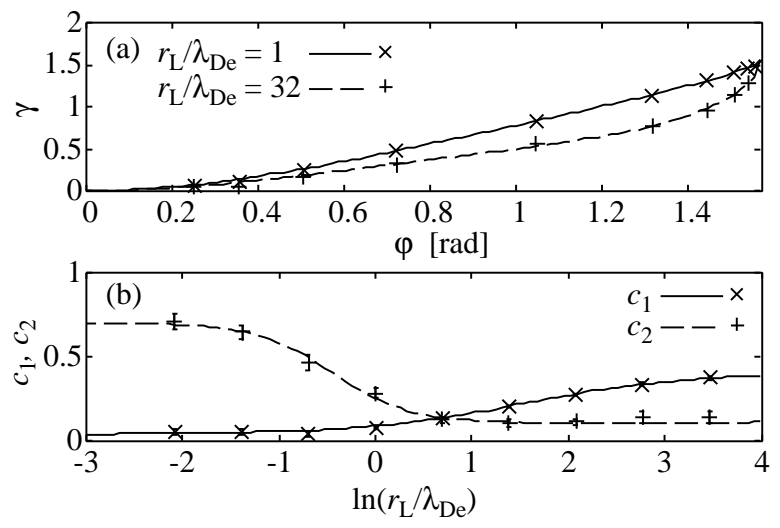

FIG. 5: Dependences of the parameters $\gamma, c_{1}$ and $c_{2}$. Curves represent fitting functions.

new parameters $c_{1}$ and $c_{2}$ are plotted in Fig. 5(b) and also characterized by the hyperbolic tangent. The transition appears in the range of $-2<\ln \left(r_{\mathrm{L}} / \lambda_{\text {De }}\right)<2$.

In summary, the normalized energy flux is written as Eq. (2) and the parameters $\beta$ and $\gamma$ are given by

$$
\begin{aligned}
\beta= & 7.2 \exp \frac{\left\{0.34+0.30 \tanh \left[1.2\left(\ln \left(r_{\mathrm{L}} / \lambda_{\mathrm{De}}\right)-2.1\right)\right]\right\} \varphi^{2}}{\left.2.0+0.38 \tanh \left[1.2\left(\ln \left(r_{\mathrm{L}} / \lambda_{\mathrm{De}}\right)-2.1\right)\right)\right]-\varphi}, \\
\gamma= & \frac{\pi}{2}(1-\cos \varphi)-\varphi^{2} \sqrt{\frac{\pi}{2}-\varphi}\left\{\left[0.22+0.18 \tanh \left(0.64\left(\ln \left(r_{\mathrm{L}} / \lambda_{\mathrm{De}}\right)-1.5\right)\right] \varphi\right.\right. \\
& -\left[0.41-0.28 \tanh \left(1.3\left(\ln \left(r_{\mathrm{L}} / \lambda_{\mathrm{De}}\right)-0.37\right)\right]\left(\frac{\pi}{2}-\varphi\right)\right\} .
\end{aligned}
$$

The constant $\alpha$ in Eq. (2) is a normalization factor. The total energy flux, i.e. $\int_{0}^{\pi / 2} Q d \theta$, is proportional to $B_{x} / B$ in our simulation because we fixed the electron temperature and changed $B_{x} / B$ and $B$ only. Although these results were obtained from the PIC simulation for $1 / 8<r_{\mathrm{L}} / \lambda_{\mathrm{De}}<32$, they can be applied for the case of weaker or stronger magnetic field because saturation of parameter $\beta$ and $\gamma$ occurs (see Figs. 4(b), (c) and 5(b)). The fitting form, Eqs. (2) - (4), can provide the $\theta$ distribution for asymptotic cases such as $\varphi=\pi / 2$ and $r_{\mathrm{L}}=0$. This is a great advantage over PIC simulation because vast amount of simulation time is required for such cases. 


\section{Conclusions}

We have presented a particle simulation model of the magnetic presheath and carried out the simulation using this model to reveal the effect of the magnetic field on the ion incident angle to the plasma-facing surface in fusion devices. In the model, different characteristics in the plasma sources of the magnetic presheath are included; the thermally equilibrium component due to the cross-field diffusion in the SOL and the ionization one in the collisional presheath region. We adopted Eq. (1) as the velocity distribution function at the entrance of the magnetic presheath.

We have examined the dependence of the ion incident angle distribution on the magnetic field. In order to characterize it, a fitting function, Eq. (2), was introduced for the energy flux as a function of the incident angle. The free parameters in Eq. (2) were given as functions of magnetic field strength and direction. They indicate an existence of a transition in the range of $-2<\ln \left(r_{\mathrm{L}} / \lambda_{\mathrm{De}}\right)<2$. The $\theta$ distribution changes only when the magnetic field strength is in the range. The width and peak position of the $\theta$ distribution depends on both of the strength and direction of the magnetic field. When the magnetic field is nearly parallel to the surface, the incident angle are large and the width of its distribution is small. In the limit of $\varphi=0$ and $\pi / 2$, the dependences on $r_{\mathrm{L}} / \lambda_{\text {De }}$ vanish.

\section{Acknowledgments}

The authors would like to thank Dr. T. Takizuka of Japan Atomic Energy Agency for helpful comments. This work was supported in part by a Grant-in-Aid for scientific research from Ministry of Education, Science and Culture of Japan (No. 19055005).

[1] T. Ono, K. Shibata, T. Kenmotsu, T. Muramoto, Z. Li and T. Kawamura, J. Nucl. Mater. 363-365 (2007) 1266

[2] D. Tskhakaya and S. Kuhn, Plasma Phys. Control. Fusion 47 (2005) A327

[3] Peter C. Stangeby, The Plasma Boundary of Magnetic Fusion Devices, Institute of Physics Publishing (Bristol and Philadelphia 1999)

[4] J. P. Gunn, Phys. Plasmas 4 (1997) 4435

[5] A. B. DeWald, A. W. Bailey and J. N. Brooks, Phys. Fluids 30 (1987) 267

[6] R. Chodura, Phys. Fluids 25 (1982) 1628

[7] R. Smirnov, Y. Tomita, T. Takizuka, A. Takayama and Yu. Chutov, Contrib. Plasma Phys., 44 (2004) 150

[8] L. Tonks and I. Langmuir, Phys. Rev. 34 (1929) 876

[9] E. R. Harrison and W. B. Thompson, Proc. Phys. Soc. 74 (1959) 145

[10] K.-U. Riemann, Phys. plasmas 13 (2006) 063508

[11] K-U Riemann, J. Phys. D: Appl. Phys. 36 (2003) 2811 